\title{
EVALUACIÓN POSCOSECHA DE LAS CARACTERÍSTICAS DEL LULO (Solanum quitoense) CUBIERTO CON HOJA DE PLÁTANO
}

\author{
POSTHARVEST EVALUATION OF LULO CHARACTERISTICS \\ COVERED WITH BANANA LEAF
}

\author{
Nathalia M. Forero', Sebastián Gutiérrez¹, Rafael L. Sandoval'1 Jesús H. Camacho*2, \\ Miguel A. Meneses ${ }^{3}$.
}

Recibido para publicación: Febrero 20 de 2014 - Aceptado para publicación: Mayo 13 de 2014

\begin{abstract}
RESUMEN
En Colombia, la producción de lulo (Solanum quitoense) es cercana a $65000 \mathrm{t}$ año ${ }^{-1}$, de las cuales se pierde cerca del $45 \%$ en la etapa poscosecha (Asociación Hortofruticola de Colombia 2006). Por esto, uno de los retos propuestos en el Plan Frutícola Nacional del 2006 es mejorar las técnicas poscosecha empleadas actualmente. El presente estudio tuvo como objetivo evaluar la hoja de plátano como empaque no convencional para el manejo poscosecha del lulo. Se observó la evolución del peso, pH, acidez titulable (AT), sólidos solubles totales (SST), intensidad respiratoria (IR) y cambios de color en cuatro tratamientos de lulo que consistieron en producto almacenado a temperatura ambiente y a $7,5^{\circ} \mathrm{C}$ con y $\sin$ hoja de plátano como empaque. El estudio consistió de un diseño al azar, realizando análisis de varianza y comparación de medias. El almacenamiento de lulo con hojas de plátano redujo las pérdidas de peso para temperatura ambiente del $11,8 \%$ al $8,3 \%$ y para producto refrigerado de $6,4 \%$ a $4,6 \%$. Se encontró que el tiempo incide en los valores de AT y SST y en cuanto al color se determinó que el cambio de verde a naranja es más tardío en el producto cubierto con hoja de plátano.
\end{abstract}

Palabras Clave: Conservación de frutas, empaque, almacenamiento, maduración.

\begin{abstract}
In Colombia, production of lulo (Solanum quitoense) is close to $65,000 \mathrm{t} \mathrm{yr}^{-1}$, which lost about $45 \%$ in the post-harvest stage (Horticulture Association of Colombia 2006). Therefore, one of the challenges proposed in the National Fruit Plan 2006 is to improve post-harvest techniques currently employed. The aim of this study was to evaluate the banana leaf as non conventional packaging for postharvest handling of lulo. For this, the evolution of weight, $\mathrm{pH}$, titratable acidity (TA), total soluble solids (TSS), respiratory rate (RR) and color changes were observed in four treatments which consisted in product storage at room temperature and

${ }^{1}$ Estudiante de Ingeniería Agrícola, Universidad Nacional de Colombia, Bogotá, Colombia.

${ }^{2 *}$ Ingeniero Agrícola, M.Sc. Ph. D., Profesor Asociado Universidad Nacional de Colombia, Bogotá, Colombia. Cra. 30 \#45-03 Ed. $214-$ 306. Telefono: 57+1+3165430 Ext. 1661. e-mail: jhcamachot@unal.edu.co.

${ }^{3}$ Ingeniero Agrícola, Profesor de cátedra, Universidad Nacional de Colombia, Bogotá, Colombia.
\end{abstract}


$7.5^{\circ} \mathrm{C}$ and with and without banana leaf as packaging. The study considers a random design, an ANOVA and comparison of means were made. The storage of lulo with banana leaves reduces the product weight losses at room temperature from $11.8 \%$ to $8.3 \%$, and for refrigerated product from $6.4 \%$ to $4.6 \%$. It was found that time affects the values of TA and TSS, and it was determined that the color change from green to orange is later in the product packed with banana leaf.

Key-words: Fruit conservation, packaging, storage, ripening.

\section{INTRODUCCIÓN}

En Colombia, la producción de lulo (Solanum quitoense) es cercana a 65000 t año-1, donde el $74 \%$ del área sembrada corresponde a la economía campesina tradicional (Criollo 2013). El lulo es un fruto climatérico (Monteiro 2013), situación por la cual es necesario garantizar un buen manejo poscosecha, que permita conservar en mayor medida las condiciones obtenidas en campo, para disminuir las pérdidas que en el caso de Colombia pueden ser cercanas a la mitad de la producción (Asociación Hortofrutícola de Colombia 2006).

Por lo tanto, las investigaciones en las técnicas poscosecha son imprescindibles en un país como Colombia, donde las pérdidas en frutas son muy altas y alcanzan valores que varían entre 10 y $30 \%$ de la producción, frente a naciones desarrolladas donde estas no sobrepasan el $8 \%$ (Gallego et al. 2003).

Algunas de las estrategias más utilizadas en países del trópico para conservar frutas son la refrigeración y el uso de atmósferas controladas (FAO 1989), las cuales permiten retardar los eventos fisiológicos y bioquímicos que llevan a la senescencia del fruto (Chitarra 2008). De esta forma, se favorece al aumento de la vida en anaquel del mismo (Kader 2002).

De igual manera, el empaque resulta otra alternativa eficiente en la poscosecha de frutas.
Su objetivo es la preservación y protección principalmente de alimentos y materias primas (Villada et al. 2007). No obstante, no solo se debe caracterizar por servir de protección al fruto si no que al ser desechado su degradabilidad sea alta. Es por esto que un empaque que se puede degradar en $\mathrm{CO}_{2}$, agua y posteriormente en abono orgánico puede ofrecer una ventaja frente a los empaques sintéticos (Bastioli 2001).

Históricamente en Colombia se han utilizado diferentes hojas como empaques con alta degradabilidad para envolver alimentos crudos y transformados, para proteger y preservar cosechas (Díaz 2014). Entre los empaques con estas características se destaca la hoja de plátano (Musa paradisiaca) y la hoja de bijao (Heliconia bihai). Este último es empleado especialmente para comercializar bocadillo veleño o bocadillo de guayaba, ya que este empaque le aporta unas características de sabor y olor agradables (Prada et al. 2006). Sin embargo, no se tiene reporte de la utilización de la hoja de plátano ni de la hoja de bijao como empaques para frutas.

En esta investigación se optó por este tipo de empaque para evaluar su efecto en el lulo, por ser un material con alta degradabilidad, de fácil acceso por ser un producto del deshoje de cultivos de plátano que se desarrollan en zonas cercanas donde se encuentra el cultivo de lulo, y puede presentar ventajas como empaque para frutas similares a las que ofrece la hoja de bijao como empaque para bocadillo veleño. 


\section{MATERIALES Y MÉTODOS}

La investigación fue realizada en el Laboratorio de Poscosecha de Frutas y Hortalizas de la Universidad Nacional de Colombia, sede Bogotá. Se tomó una muestra de 20 kg (196 unidades) de fruto con buena apariencia, variedad quitoense, de la zona productora de Cundinamarca. El estudio se basó en un diseño al azar con arreglo factorial de $2 * 2$ y cuatro repeticiones por tratamiento, con frutos tomados aleatoriamente para cuatro tratamientos: a) ambiente con hoja de plátano cubriendo el lulo $(\mathrm{ACH})$; b) ambiente sin hoja de plátano $(\mathrm{ASH}) ; \mathrm{c})$ refrigerado con hoja de plátano cubriendo el lulo $(\mathrm{RCH})$ y d) refrigerado sin hoja de plátano (RSH). Las condiciones de temperatura trabajadas fueron ambiente de $18^{\circ} \mathrm{C}$ y refrigeración de $7,5 \pm 0,5^{\circ} \mathrm{C}$. El almacenamiento en todos los tratamientos consistió en hojas de plátano que envolvían entre 5 y 6 lulos en hileras, las cuales fueron acomodadas en cajas de plástico y situadas en la nevera o en un estante según correspondiera. Con el fin de evitar la propagación de hongos provenientes de la hoja de plátano, esta fue tratada con agua a $70^{\circ} \mathrm{C}$.

Durante 20 días se realizó el seguimiento de la pérdida de peso $(\mathrm{Pp})$, intensidad respiratoria (IR) cambio de color, $\mathrm{pH}$, acidez titulable (AT), sólidos solubles totales (SST) y relación de madurez. Esta evaluación se realizó cada tres días para los cuatro frutos de cada tratamiento. El pH fue determinado con un medidor Mettler, la IR se midió con una cámara de respiración Vernier, que registra la concentración de $\mathrm{CO}_{2}$ en intervalos de 5 minutos, los SST fueron medidos con un refractómetro manual óptico $\mathrm{SO}-\mathrm{RH}$ en grados Brix, la AT se expresó como porcentaje de ácido cítrico y el color se midió con ayuda de un Colorímetro digital "Konica Minolta CR-410", registrando las medidas en las coordenadas del modelo cromático $L^{*}\left(\right.$ Luminosidad); $a^{*}$ y $b^{*}$ (Coordenadas de cromaticidad).

Un parámetro indirecto que se determinó fué la relación de madurez, la cual se define por la Ecuación (1).

\section{Relación de madurez $=\frac{S S T}{A T}$}

Con los resultados se realizó análisis de varianza y comparación de medias, mediante la prueba de Duncan, usando el paquete estadístico IBM SPSS Statistics 20.

\section{RESULTADOS Y DISCUSIÓN}

Para esta investigación, la perdida de peso (Pp) no muestra diferencias considerables entre los tratamientos $\mathrm{ACH}$ y $\mathrm{RSH}$. Al comparar resultados de las condiciones no refrigeradas, ACH permite obtener un nivel de conservación del peso más alto que ASH (Figura 1).

Luego de cosechadas, la mayoría de las frutas contiene entre el $80 \%$ y el $95 \%$ de agua por peso (Pontin et al. 2005). Como consecuencia de la transpiración, se pierde parte de este contenido de agua de los tejidos vivos, lo que representa una disminución de la calidad del fruto. Por eso el control de la pérdida de peso se vuelve indispensable para el almacenamiento de cualquier fruta.

El peso presenta diferencias significativas entre la temperatura de almacenamiento, el día de almacenamiento y el tipo de empaque. A 


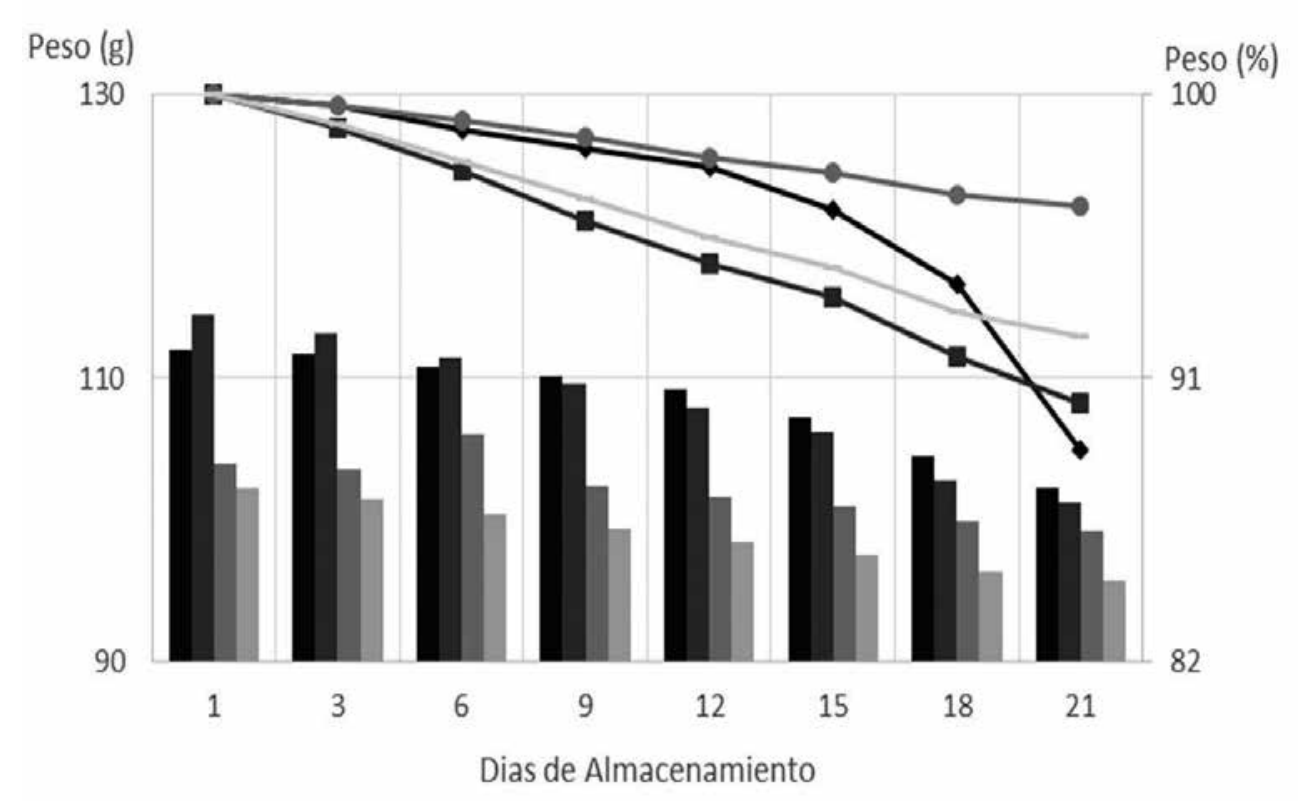

$\mathrm{ACH}$

ASH

$\mathrm{RCH}$ RSH

$\multimap \% \mathrm{ACH}$

Figura 1. Variación del peso en los cuatro tratamientos utilizados en la investigación

diferencia de los demás, el tratamiento $\mathrm{ACH}$ no tiene un comportamiento lineal desde el día 12 de almacenamiento (Figura 1), lo cual puede ser explicado por una alteración de tipo biológico causada por la interacción entre el lulo y la hoja de plátano.

Para las muestras almacenadas a temperatura ambiente, se registraron pérdidas a los 15 días de $7,3 \%$ para frutos sin empaque y de $3,8 \%$ para frutos con empaque. Dicho resultado es inferior al reportado por Balaguera et al. (2014), el cual corresponde al 12,9\% para el mismo intervalo de tiempo. Para el lulo de la experiencia refrigerado, con RSH la pérdida es del $5,8 \%$ y para $\mathrm{RCH}$ la pérdida equivale al 4\% (Figura 1), ambos a los 18 días, similares a los reportados por Reina et al. (1998) que trabajó Solanum quitoense en condiciones de refrigeración a $12^{\circ} \mathrm{C}$, con una pérdida de peso del $7,7 \%$ a los 18 días.
Con empaques convencionales (cajas de madera y de cartón) se encuentra que sin refrigerar la pérdida media de peso al décimo día es de 6,8\% y 7,1\% para empaques de madera y cartón respectivamente. Por otro lado, para lulo refrigerado se encuentra que la pérdida media de peso es menor. Bonilla (2010) reporta que al décimo día, la pérdida de peso equivale al $1,9 \%$ y $4,2 \%$ para empaques de madera y cartón respectivamente. En comparación a las pérdidas registradas con lulo almacenado en estos empaques, en el presente estudio se encontró que la pérdida media de peso al noveno día es de 1,7\% y 1,5\% para el tratamiento $\mathrm{ACH}$ y $\mathrm{RCH}$ respectivamente, lo cual muestra un comportamiento equiparable con empaques desarrollados industrialmente. La hoja de plátano además de mostrar que cumple su función de reducir las pérdidas de peso por transpiración, lo cual se demuestra con base en los resultados anteriores, también 
ofrece otras ventajas como su bajo costo, su posibilidad de ser empaque individual y sobre todo su biodegradabilidad, ventaja comparativa con respecto a polímeros sintéticos usados en el empacado de alimentos (Villada et al. 2007). La razón por la cual la pérdida de peso es menor en los productos empacados en hoja de plátano, se atribuye a que esta funciona como barrera para las corrientes de aire, las cuales, tanto en almacenamiento refrigerado como en almacenamiento no refrigerado, pueden tener humedades relativas más bajas que el contenido de humedad de la fruta.

El desequilibrio entre la humedad de la fruta y la humedad del ambiente genera un fenómeno en el cual se transfiere la humedad de la fruta al ambiente para equilibrar la humedad del sistema. Debido a que la hoja funciona como barrera, es esta la que pierde humedad y en menor medida el fruto, efecto que se evidencio durante la experiencia.
El empaque de hoja de plátano resulta tener incidencia sobre los valores de $\mathrm{pH}$, sin embargo, no hay una tendencia clara en el comportamiento de esta variable. Diferentes investigaciones indican que el $\mathrm{pH}$ es un parámetro que varía durante el proceso de maduración de frutos climatéricos (Novoa et al. 2006; Fernandes et al. 2013; Ferreira et al. 2013), y también es afectado por la temperatura de almacenamiento (Parra et al. 2006).

Cabe resaltar que los valores que se obtuvieron no presentan diferencias apreciables entre tratamientos para un mismo día (Tabla 1), lo que indica que el $\mathrm{pH}$ no es una variable relevante en el proceso de maduración del lulo, tal como lo afirman otros autores (Pinzón 2000; Casierra et al. 2004).

El color presentó diferencias significativas respecto al empaque y al tiempo de almacenamiento. Los dos tratamientos

Tabla 1. Valores medios de $\mathrm{pH}$ del lulo bajo los cuatro tratamientos manejados.

\begin{tabular}{lcccccccc}
\hline & & ACH & & ASH & & RCH & RSH \\
\cline { 2 - 8 } & Media & & Media & & Media & & Media \\
\hline 1 & 3,07 & a & 3,26 & abcdef & 3,12 & abc & 3,22 abcd \\
3 & 3,29 & bcdef & 3,44 & efg & 3,24 & abcdef & 3,25 abcdef \\
6 & 3,25 & abcdef & 3,26 & abcdef & 3,35 & def & 3,26 abcdef \\
9 & 3,26 & abcdef & 3,12 & ab & 3,19 & abcd & 3,24 abcdef \\
12 & 3,39 & defg & 3,30 & bcdef & 3,32 & bcdef & 3,46 & fg \\
15 & 3,36 & defg & 3,44 & efg & 3,28 & abcdef & 3,24 abcdef \\
18 & 3,44 & efg & 3,34 & cdef & 3,37 & defg & 3,38 defg \\
21 & 3,24 & abcde & 3,19 & abcd & 3,34 & def & 3,57 & g \\
\hline & & & & & & & &
\end{tabular}

Medias seguidas de letras distintas indican diferencias, según prueba de Duncan $(P<0.05)$.

$\mathbf{A C H}=$ Ambiente con hoja de plátano $\mathbf{A S H}=$ Ambiente sin hoja de plátano

$\mathbf{R C H}=$ Refrigerado con hoja de plátano $\mathbf{R S H}=$ Refrigerado con hoja de plátano 


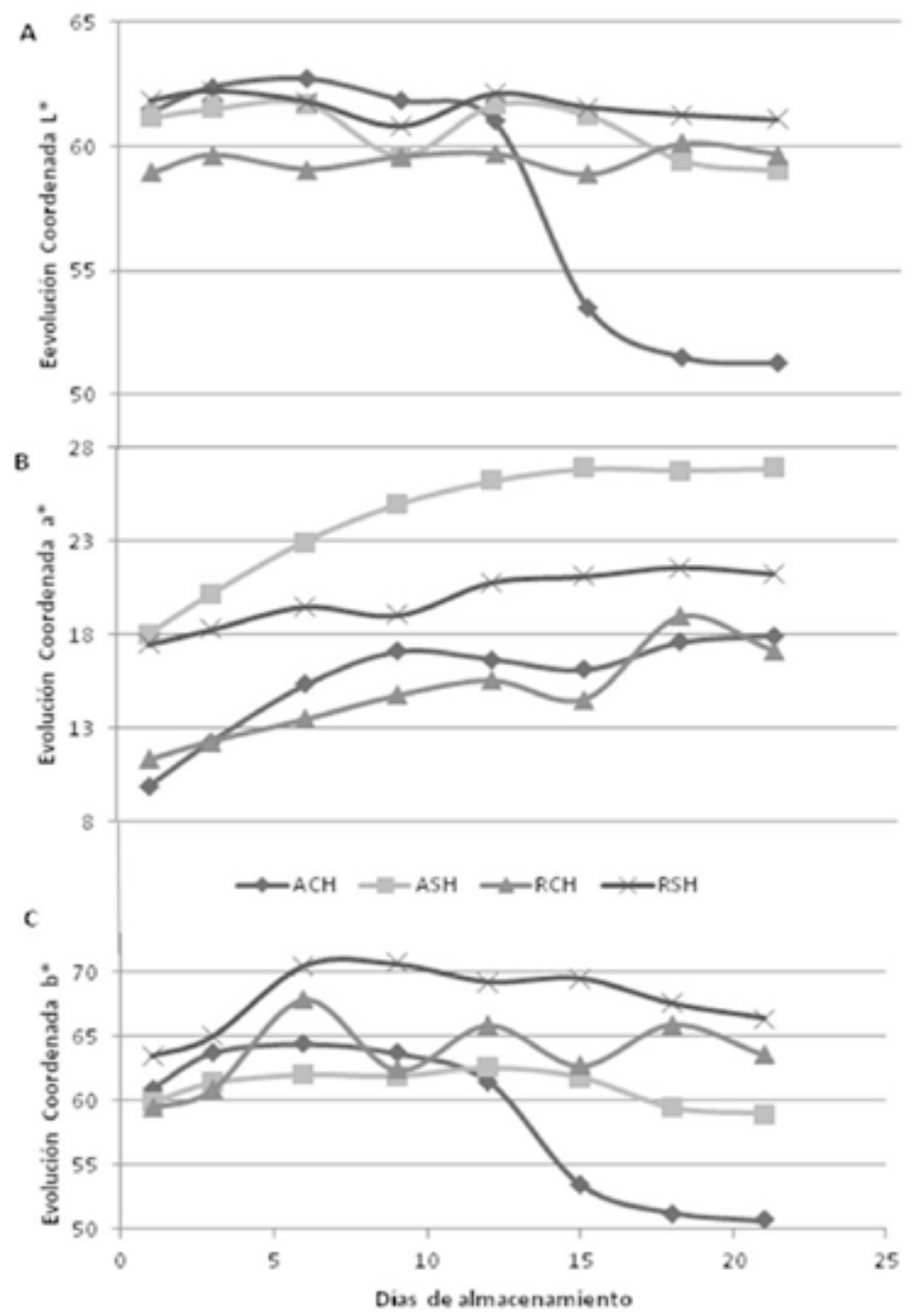

Figura 2. Evolución del color durante el tiempo de almacenamiento, evolución coordenada $L^{*}(A)$, evolución coordenada a* (B) y evolución coordenada b*(C).

refrigerados mostraron un cambio de color más uniforme en comparación con el producto almacenado a temperatura ambiente (Figura 2).

Se partió de la hipótesis de que el lulo pasa de una tonalidad verde - amarilla a una amarilla - naranja (Pinzón 2000). Se observó que el valor de las coordenadas $L^{*}$ y $b^{*}$ no tienen una tendencia clara, mientras que $a^{*}$ aumenta en la medida que el proceso de maduración del lulo avanza. Para la coordenada a* (cambios de color de verde a rojo) se observa que en los tratamientos con hoja el cambio es más tardío que en los tratamientos sin hoja (Figura 2B). En la coordenada b* (cambios de color de amarillo a azul) todos los tratamientos evolucionan hacia una tonalidad amarilla lo que explica el color representativo del lulo (Figura 2C).

Lo anteriormente dicho se debe a la pérdida de la clorofila propia del proceso de maduración, donde la degradación de este pigmento provoca una síntesis de carotenoides dando paso a coloraciones naranjas y amarillas. (Salinas et al. 2010). Las condiciones de baja temperatura juegan un papel importante 
en la estabilidad de los pigmentos del fruto desacelerando el proceso de maduración. Igualmente el empaque protege el fruto del ambiente y disminuye la cantidad de oxigeno circundante disponible para el Lulo (Salinas et al. 2010). Es por esto que podría decirse que ambas condiciones provocan una aparición de los colores naranjas y amarillos más tardía.

Dicho cambio de coloración resulta ser un parámetro para determinar el estado de madurez para frutos de la familia solanácea, para estos el cambio de coloración se da de verde a rojo, naranja o amarillo (FAO 2002; Uroz 2012).

Es importante resaltar el comportamiento que se presentó a partir del día 12 para el tratamiento $\mathrm{ACH}$, en el cual hubo un descenso significativo con respecto a los demás tratamientos para las coordenadas $L^{*} y b^{*}$. Esto coincide con la alteración observada para el mismo tratamiento en la variable de pérdida de peso (Figura 2A y 2C).

Tabla 2. Valores medios de SST y de AT (\% Ácido Cítrico) del lulo bajo los cuatro tratamientos manejados.

\begin{tabular}{|c|c|c|c|c|c|c|c|}
\hline \multirow{2}{*}{ Día } & \multicolumn{3}{|c|}{$\mathrm{ACH}$} & \multirow[t]{2}{*}{ ASH } & \multicolumn{2}{|r|}{$\mathbf{R C H}$} & RSH \\
\hline & Media & & Media & & Media & & Media \\
\hline & & & & SST & & & \\
\hline 1 & 8,00 & cdefghij & 8,63 & efghij & 8,00 & cdefghij & 8,25 defghij \\
\hline 3 & 7,86 & cdefghij & 9,33 & j & 8,86 & hij & $9,30 \quad J$ \\
\hline 6 & 8,50 & efghij & 8,28 & defghij & 9,13 & ij & 8,65 efghij \\
\hline 9 & 8,33 & defghij & 8,70 & fghij & 7,70 & cdefghi & 8,23 defghij \\
\hline 12 & 7,33 & abcdefg & 6,98 & abcd & 6,25 & $a b$ & 6,73 abc \\
\hline 15 & 7,25 & abcdef & 7,68 & cdefghi & 8,35 & defghij & 7,38abcdefgh \\
\hline 18 & 7,53 & bcdefgh & 8,40 & defghij & 9,15 & $\mathrm{ij}$ & 8,78 ghij \\
\hline 21 & 6,06 & a & 7,18 & abcde & 7,20 & abcde & 7,80 cdefghi \\
\hline \multicolumn{8}{|c|}{ AT } \\
\hline 1 & 3,85 & g & 3,39 & defg & 3,54 & fg & 3,89 \\
\hline 3 & 2,86 & abcdef & 3,05 & bcdefg & 2,58 & abcd & 2,81 abcdef \\
\hline 6 & 2,86 & abcdef & 3,46 & efg & 3,12 & bcdefg & 3,54 \\
\hline 9 & 2,15 & $\mathrm{a}$ & 2,43 & abc & 2,78 & abcdef & 3,36 defg \\
\hline 12 & 2,59 & abcde & 2,87 & abcdef & 3,35 & defg & 2,81 abcdef \\
\hline 15 & 2,77 & abcdef & 2,82 & abcdef & 2,91 & abcdef & 3,18 cdefg \\
\hline 18 & 2,54 & abcd & 2,66 & abcde & 3,13 & bcdefg & 3,46 \\
\hline 21 & 2,31 & $a b$ & 2,74 & abcdef & 2,70 & abcdef & 2,18 \\
\hline
\end{tabular}

Medias seguidas de letras distintas indican diferencias, según prueba de Duncan $(P<0,05)$.

$\mathbf{A T}=$ Acidez titulable $\quad \mathbf{S S T}=$ Solidos solubles totales

$\mathbf{A C H}=$ Ambiente con hoja de plátano $\mathbf{A S H}=$ Ambiente sin hoja de plátano $\mathbf{R C H}=$ Refrigerado con hoja de plátano $\mathbf{R S H}=$ Refrigerado con hoja de plátano 
Vale la pena destacar que el color juega un papel significativo en la comercialización del lulo, pues ciertos consumidores buscan que el fruto tenga su color característico (amarillonaranja), por lo tanto la hoja de plátano resulta ser una alternativa favorable en este caso.

Los sólidos solubles totales (SST) presentaron diferencias significativas entre las configuraciones del empaque y entre el tiempo de almacenamiento (Tabla 2).

En frutas tropicales se registra que los SST aumentan con el transcurso del proceso de maduración (Magaña et al. 2004; Parra et al. 2006; Almeida et al. 2011) y luego decrece.

Se observó que el incremento significativo en los frutos evaluados con empaque se da del tercer al sexto día, mientras que éste incremento para los frutos sin empaque se presenta entre el primer y el tercer día.

Galvis y Herrera (1999) reportan que los SST en lulo varían de 7,8 a 9,3Brix; y Casierra et al. (2004) obtuvo variaciones de 8 a $10^{\circ}$ Brix. Estos resultados son similares a los que se obtienen en la presente investigación los cuales varían de 8 a $9,3^{\circ}$ Brix y luego decrece drásticamente hasta registrar valores de $7^{\circ} \mathrm{Brix}$.

Sin embargo, una de las causas importantes que puede generar variación en la concentración de SST es el grado de madurez con el cual fue cosechado el fruto (Casierra et al. 2004). Frutos cosechados en un estado de madurez mayor, tendrán una mayor concentración de SST.

La temperatura de almacenamiento, el tipo de empaque y el día de almacenamiento inciden sobre la AT. Para los cuatro tratamientos manejados, el porcentaje de ácidos cítricos disminuye a medida que el lulo madura, sin conservar una tendencia lineal (Tabla 2).

A su vez, el uso de la hoja de plátano como empaque incide en este valor como se observa en la tabla 2, ya que generalmente el valor de AT fue mayor en los frutos sin hoja de plátano. La tendencia de las muestras refrigeradas es similar a lo encontrado por Pinzón (2000), que en su investigación reporta que la concentración total de ácidos orgánicos se incrementa del cuarto al sexto día de almacenamiento a $7^{\circ} \mathrm{C}$ y luego decrece drásticamente.

Las diferencias significativas con el paso del tiempo y la temperatura de almacenamiento también fueron reportadas por Casierra et al. (2004). La temperatura de almacenamiento adecuada en lulo tiene un papel importante no solo para conservar peso sino para conservar otras propiedades como es el porcentaje de ácido cítrico.

En respuesta a las anteriores variables, la relación de madurez (SST/AT) para todos los tratamientos, es mayor al final del proceso de maduración. No se observa una tendencia clara, sin embargo esta relación permanece más baja en frutos refrigerados entre el noveno y doceavo día (Figura 3). Este parámetro, a pesar que está relacionado con la evolución del proceso de maduración, no es un parámetro adecuado para diferenciar el comportamiento del lulo entre tratamientos; ya que con una misma relación pueden variar los componentes químicos implícitos en esta ecuación (Acevedo 2008). 
El comportamiento de la relación de madurez en la presente investigación obedece a su misma definición. Por lo tanto, es la resultante de la reducción de la AT y del aumento en los SST, a medida que el proceso de maduración avanzaba. Este comportamiento también es reportado en lulo (Casierra et al. 2004) y tomate (Casierra et al. 2008).

No existe relación directa entre la intensidad respiratoria, el tiempo de almacenamiento, el tipo de empaque ni la temperatura de almacenamiento. La IR fue superior en los tratamientos que no fueron refrigerados. La temperatura de refrigeración cumplió su función de retardar la senescencia. A diferencia de la Pp y las coordenadas de color $\mathrm{L}^{*}$ y $\mathrm{b}^{*}$, el tratamiento $\mathrm{ACH}$ no presenta un comportamiento diferente a los demás en el día 12. En general la mayor tasa de respiración se presentó en el tratamiento $\mathrm{ACH}$ lo cual puede ser causado por su interacción con la hoja de plátano. En la Figura 3 es posible ver que el aumento de la intensidad respiratoria se dio a partir de la segunda lectura. Este comportamiento indica que el producto llegó a la central de abasto con avanzado estado de maduración, pues este aumento es normal en frutos climatéricos en este avanzado estado (Dussan et al. 2008; Trujillo et al. 2010).

La intensidad respiratoria se mantuvo en toda la experiencia entre 5 y $40 \mathrm{mg}$ de $\mathrm{CO}_{2} \mathrm{~kg}^{-1} \mathrm{~h}^{-1}$ para todos los tratamientos. Estos iniciaron con valores de intensidad respiratoria similar, sin embargo, esta variable aumentó a través del

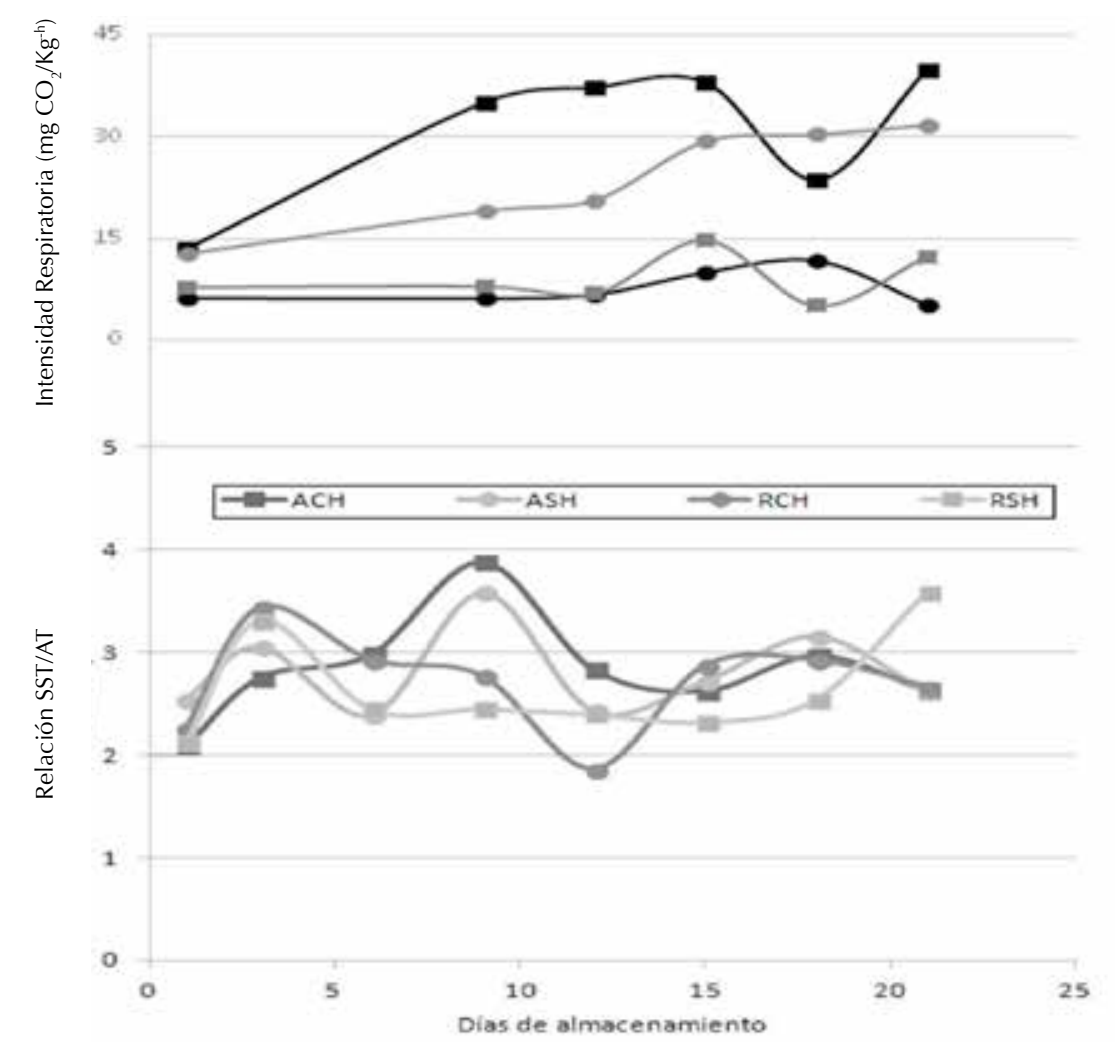

Figura 3. Evolución de la producción de $\mathrm{CO}_{2}$ y de la relación de madurez a lo largo de los días de almacenamiento para los cuatro tratamientos. 
tiempo para los tratamientos no refrigerados. La intensidad respiratoria no superó los $15 \mathrm{mg}$ de $\mathrm{CO}_{2} \mathrm{~kg}^{-1} \mathrm{~h}^{-1}$ para los tratamientos refrigerados, en contraste los tratamientos no refrigerados nunca estuvieron por debajo de $18 \mathrm{mg}$ de $\mathrm{CO}_{2}$ $\mathrm{kg}^{-1} \mathrm{~h}^{-1}$ desde el sexto día de almacenamiento. La hoja de plátano no generó efecto alguno sobre los frutos almacenados.

La hoja de plátano además de ser utilizada por su atractivo para exhibir algunos productos, en este caso cumple su función como empaque al conservar y proteger el lulo. Como consecuencia natural del uso de este empaque, hay un enriquecimiento de $\mathrm{CO}_{2}$ (Lemus 1999) y así mismo una reducción de la cantidad de oxigeno disponible alrededor del lulo empacado, lo cual retarda su evolución en cuanto al color. Además de esto, la hoja de plátano como empaque primario es un aislamiento térmico para reducir la pérdida de peso.

\section{CONCLUSIONES}

La hoja de plátano como empaque para el lulo es una alternativa eficiente pues genera beneficios en peso equiparables a empaques comerciales. Usar la hoja de plátano como empaque genera pérdidas de peso más bajas que el no usarla, tanto en frutos refrigerados como en frutos almacenados a temperatura ambiente.

La hoja de plátano como empaque aumenta la concentración de $\mathrm{CO}_{2}$ en cercanías al fruto, lo que resulta en una disminución de $\mathrm{O}_{2}$ y un retraso en el cambio de color de verde a naranja.

No se evidencia un efecto notable del empleo de hoja de plátano como empaque para el lulo en las variables químicas SST, AT, $\mathrm{pH}$ y SST/AT.
El empaque de hoja de plátano puede incidir en el sabor ácido representativo del lulo, representado en la variable AT.

Al igual, la temperatura es un factor que retarda el proceso de maduración, e incide en la variable de AT.

\section{REFERENCIAS}

Acevedo, Y. 2008. Eventos fisiológicos asociados a la madurez y calidad de frutos cítricos en Cuba y su relación con los productos transformados de la industria. http://www.fao.org/docs/ eims/upload/cuba/1067/cuf0114s.pdf [9 Septiembre 2014].

Almeida, A., Reis, J., Santos, D., Vieira, T. y Oliveira, M. 2011. Estudio de la conservación de la papaya (Carica papaya L.) asociado a la aplicación de películas comestibles. Revista Venezolana de Ciencia y Tecnología de Alimentos. 2(1): 49-60.

Asociación Hortofruticola de Colombia. 2006. Plan Frutícola Nacional. http://www. asohofrucol.com.co/archivos/biblioteca/ biblioteca_18_DIAGNOSTICO\%20 FRUTICOLA\%20NACIONAL.pdf [24 Febrero 2014].

Balaguera, H., Ramirez, D. y Almanza, P. 2014. El tiempo de inmersión en $\mathrm{CaCl} 2$ y la refrigeración modifican algunas características físicas del fruto de lulo (Solanum quitoense Lam.) durante la poscosecha. Revista Postharvest and quality management of horticultural 
products of interest for Tropical Regions.1016: 147-150.

Bastioli, C. 2001. Global status of the production of biobased packaing materials. Starch/ Stärke. 53: 351-355.

Bonilla, D. 2010. Evaluación de dos índices de cosecha y tipos de empaque del fruto de naranjilla (Solanum quitoense Lam.) Híbrido INIAP Palora, bajo dos condiciones de almacenamiento, en el cantón cevallos de la provincia de Tungurahua. Tesis Ingeniero Agrónomo, Universidad Técnica De Ambato, Cevallos.

Casierra, F. y Aguilar, O. 2008. Calidad en frutos de tomate (Solanum lycopersicum L.) cosechados en diferentes estados de madurez. Revista Agronomía Colombiana. 26(2): 300-307.

Casierra, F., Garcia, E. y Ludders, P. 2004. Determinación del punto óptimo de cosecha en el lulo (Solanum quitoense Lam. Var. Quitoense y septentrionale). Revista Agronomía Colombiana. 22(1): 32-39.

Chitarra, S. 2008. Qualidade de pimentao armazenado sob refrigeracao em atmosfera modificada. Tesis Maestría en ciencia de alimentos, Universidad Federal de Lavras, Minas Gerais. 320 p.

Criollo, H. 2013. Estudios orientados a la regeneración de plantas de lulo (Solanum quitoense Lam.) a través de la embriogénesis somática. Tesis Doctorado en Ciencias Agrarias con énfasis en
Fisiología de Cultivos, Universidad Nacional de Colombia, Bogotá.

Diaz, S. 2014. Las hojas de las plantas como envoltura de alimentos. MinCultura, Bogotá. p20-88.

Dussán, S., Honório, S. y Matias, M. 2008. Resistencia mecánica, tasa respiratoria y producción de etileno de caqui 'Fuyu' durante el almacenamiento. Revista Brasilera de Ingeniería Agrícola y Ambiental. 12(5): 498-502.

FAO. 2002. Manual preparado por el Grupo de Cultivos Hortícolas: El Cultivo Protegido en Clima Mediterráneo. http://www. fao.org/docrep/005/s8630s/s8630s00. htm\#Contents [5 Mayo 2014].

Fernandes, D., Villa, F., Barp F., Copello, M. y Stumm, D. 2013. Conservação pós-colheita de fisális e desempenho produtivo em condicões edafoclimáticas de Minas Gerais. Revista Ceres. 60(6): 826-832.

Ferreira, L., Oliveira, F., Almeida, A., Bezerra, R., Loiola, A., Cabral, E., y Porto, V. 2013. Caracterização físico-química de frutos de pimentão em diferentes acessos mercadológico. Revista Agropecuária Científica no Semi-Árido. 9(1): 99-103.

Gallego, S., Riaño, C. y Orozco, L. 2003. Determinación del comportamiento químico y fisiológico de Feijoa sellowiana en almacenamiento. Cenicafé. 54(1): 50-62. 
Galvis, J. y Herrera, A. 1999. El lulo (Solanum quitoense Lam). Manejo de poscosecha. Convenio SENA. Universidad Nacional de Colombia- Instituto Colombiano de Ciencia y Tecnología de Alimentos (ICTA). Bogotá: Colombia: Publicaciones SENA. 59 p.

Kader, A. 2002. Postharvest Technology of Horticultural Crops. UCANR Publications, California. p193-203.

Lemus, E. y Ramirez, J. 1999. Modelado dinámico del intercambio de gases en el envase de frutas y vegetales bajo atmósferas modificadas. Revista Temas de Ciencia y Tecnología. 3(9): 47-50.

Magaña, W., Balbín, M., Corrales, J., Rodríguez, A., Saucedo, C., Cañizares, E. y Sauri, E. 2004. Efecto de la frigoconservación en el comportamiento fisiológico de frutas de pitahaya (Hylocereus undatus Haworth). Revista Cultivos Tropicales. 24(4): 33-39.

MinAgricultura. 2013. Anuario estadístico del sector agropecuario 2012. http:// www.agronet.gov.co/agronetweb1/ Estad\%C3\%ADsticas.aspx [3 Marzo 2014].

MinAgricultura. 2013. Sistema de estadísticas agropecuarias. http://www.agronet. gov.co/www/htm3b/ReportesAjax/ parametros/reporte $16 \_2011$. aspx?cod=16 [24 Febrero 2014].

Monteiro, L. 2013. Desenvolvimento dos frutos de lulo (Solanum quitoense Lam), em viçosa-mg. Revista Brasilera de Fruticultura. 35(1): 131-142.
Novoa, R., Bojacá, M., Galvis, J. y Fischer, G. 2006. La madurez del fruto y el secado del cáliz influyen en el comportamiento poscosecha de la uchuva, almacenada a $12^{\circ} \mathrm{C}$ (Physalis peruviana L.). Revista Agronomía Colombiana. 24(1): 77-86.

Parra, A., Hérnandez, J. y Camacho, J. 2006. Comportamiento fisiológico de la pera variedad Triunfo de Viena (Pyrus communis L.) durante el período poscosecha. Revista Bras. Frutic. Jaboticabal. 28(1): 46-50.

Pinzón, M. 2000. Propiedades físicas de cosecha y poscosecha de frutos de Lulo "La Selva". Memorias del 3er Seminario de Frutales de Clima Frío Moderado. Centro de Desarrollo Tecnológico de Frutales (CDTF), Manizales. p386-397.

Prada, L., García, H., Koop, E. y Cáceres, J. 2006. Bijao: Un empaque para la certificación de origen del bocadillo veleño. http://corpomail.corpoica.org. co/BACFILES/BACDIGITAL/41486/ Bijaounempaqueparalacertificacin.pdf [9 Septiembre 2014]

Pontin, M., Lema, A., Ritta, R., Sanmartino, A. y Ghirardotto, M. 2005. Secadero Solar para frutas: Estudio de Caso. Revista Avances en Energia y Medio Ambiente. 9: 43-48.

Reina, C., Araujo, C. y Manrique, I. 1998. Manejo postcosecha y evaluacion de la calidad del lulo que se comercializa en la ciudad de Neiva. Universidad Surcolombiana, Neiva. p86-94. 
Salinas, R., Liévano, E., Ullín, F., Mercado, J. y Jiménez, D. 2010. Caracterización morfológica y cambios durante la vida postcosecha de cuatro tipos de chile amashito (Capsicum annuum I.) variedad glabriusculum (dunal) heiser \& pickersgill. Revista Iberoamericana de Tecnología Postcosecha. 11(1): 92-100.

Trujillo, Y. y Suarez, J. 2010. Evaluación del proceso de maduración del lulo (Solanum quitoense Lam) variedad Castilla. Revista @limentech Ciencia y Tecnología Alimentaria. 8(1): 58-66.
Uroz, S. 2012. Caracterización de variedades locales de solanáceas: cuatro de tomate y tres de pimiento. Tesis Maestría en Agricultura Ecológica, Universidad de Barcelona, Barcelona.

Villada, H., Acosta, H. y Velasco, R. 2007. Biopolímeros naturales usados en empaques biodegradables. Revista Temas agrarios. 12(2): 5-13.

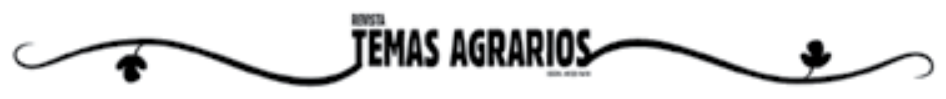

